hep-th/0106145

FIT HE - 01-02

Kagoshima HE-01-3

\title{
Massive vector trapping as a gauge boson on a brane
}

\author{
Kazuo Ghoroku円 and Akihiro Nakamuraf \\ ${ }^{1}$ Fukuoka Institute of Technology, Wajiro, Higashi-ku \\ Fukuoka 811-0295, Japan \\ ${ }^{2}$ Kagoshima University, Korimoto 1-21-35 \\ Kagoshima 890-0065, Japan
}

\begin{abstract}
We propose a mechanism to trap massive vector fields as a photon on the Randall-Sundrum brane embedded in the five dimensional AdS space. This localization-mechanism of the photon is realized by considering a brane action, to which a quadratic potential of the bulk-vector fields is added. We also point out that this potential gives several constraints on the fluctuations of the vector fields in the bulk space.
\end{abstract}

\footnotetext{
${ }^{1}$ gouroku@dontaku.fit.ac.jp

${ }^{2}$ nakamura@sci.kagoshima-u.ac.jp
} 


\section{Introduction}

People expect that our four dimensional world would be embedded in the higher dimensional space-time where the geometry would be determined by the superstring theory. The Randall-Sundrum brane-model (RS brane) [1, 2] would be a probable candidate of such a simplified theory with more extra-dimensions. It is possible to consider such that the RS brane is embedded at some point of the transverse coordinate in the fivedimensional anti-de Sitter $\left(\mathrm{AdS}_{5}\right)$ space. And $\mathrm{AdS}_{5}$ is realized near the horizon of the background geometry constructed by the stack of many D3-branes.

In order to consider this RS brane as our four-dimensional world, it would be necessary to confine all observed fields in this brane. Up to now, it is known that the gravity and scalars can be trapped on the brane of positive tension [2, 3], and fermions are localizable on the one of negative tension [4, 5, 3] due to the gravitational force coming from the background configuration, $\mathrm{AdS}_{5}$. However no one knows how the gauge bosons can be trapped on the RS brane by the same situation.

Some ideas [6, 7, 8, 9] for the localization of the gauge fields have been proposed without relating the mechanism to the gravitational force only. Another interesting mechanism has been proposed in [10], where a special mass term has been introduced in the bulk action through three-form field. As a result, the zero-mode of the photon becomes localizable on the brane. However, a resultant four-dimensional action contains a mass term of the gauge boson characterized by the introduced topological mass. In this sense, this mechanism should be modified to a more reasonable one.

The purpose of this paper is to propose a new localization-mechanism of the gauge field by considering a massive vector in the bulk and a slightly modified brane-action. The modification of the brane-action is performed by adding a localized potential of the vector fields which are living in the bulk. The idea to add such a potential of the bulk field is also seen in [11] for the case of scalar fields, and its possible origin might be found in the quantum corrections in the bulk [12]. Here the potential on the brane is given by a quadratic form of this vector field, which can be regarded as a localized mass-term of the vector. Then we can show that the zero-mode, which means zero four-dimensional mass of this mode, of the vector is localized on the brane as a gauge field. In other words, the broken gauge-symmetry in the bulk is restored on the brane of reduced dimensions. Then our mechanism would be inverse of the one given in [10], where the bulk gauge-symmetry seems to be broken on the brane due to the induced mass-term of the localized vector-field. We should point out another point that the solutions for the fluctuations of the massive vector in $\mathrm{AdS}_{5}$ background are solved by including odd functions with respect to the coordinate perpendicular to the brane.

In the next section, our localization-mechanism is shown by solving the field equations of the vector fields according to the parallel method used in [13, 14]. In the section three, this result is further assured through the study of the Green function of the vector in the bulk space. Final section is devoted to the summary. 


\section{Localized state of the vector}

Here we start from the following effective action,

$$
S=S_{\mathrm{gr}}+S_{\mathrm{A}}
$$

The first term denotes the gravitational part,

$$
S_{\mathrm{gr}}=\frac{1}{2 \kappa^{2}}\left\{\int d^{5} X \sqrt{-G}(R+\Lambda)+2 \int d^{4} x \sqrt{-g} K\right\}-\frac{\tau}{2} \int d^{4} x \sqrt{-g},
$$

where $K$ is the extrinsic curvature on the boundary, and $\tau$ represents the tension of the brane. The background configuration with RS brane,

$$
d s^{2}=e^{-2|y| / L} \eta_{\mu \nu} d x^{\mu} d x^{\nu}+d y^{2}
$$

is determined by this action, where $\eta_{\mu \nu}=(-,+, \cdots,+)$. Here $\tau=6 /\left(L \kappa^{2}\right)$ and $L=\sqrt{6 / \kappa^{2} \Lambda}$ which denotes the radius of five-dimensional AdS space. The coordinates parallel to the brane are denoted by $x^{\mu}$ and $y$ is the coordinate transverse to the brane.

The second part $S_{\mathrm{A}}$ denotes the action for the massive vector, which is denoted by $A_{M}(x, y)$

$$
\begin{aligned}
S_{\mathrm{A}}= & \int d^{5} X \sqrt{-G}\left(-\frac{1}{4} G^{M N} G^{P Q} F_{M P} F_{N Q}-\frac{1}{2} M^{2} G^{M N} A_{M} A_{N}\right) \\
& -c \int d^{4} x \frac{1}{2} \sqrt{-g} g^{M N} A_{M} A_{N}
\end{aligned}
$$

where the second integral is defined on $X^{5}=y=0$. The parameter $M$ and $c$ denote the bulk mass of the vector and the coupling of the vector potential and the brane respectively. Here no field other than the vector and gravity is considered, so we ignore to consider the origin of the mass $(M)$ of $A_{M}$ and the gauge symmetry expected before the generation of $M$ in the bulk. And this action is the starting point.

Here we concentrate our attention on the behaviors of the vector-fields fluctuation around the background (3) according to the analysis of the massive scalar given in [13]. Then the field equation of $A_{M}$ is given as

$$
\frac{1}{\sqrt{-G}} \partial_{A}\left[\sqrt{-G}\left(G^{A B} G^{C D}-G^{A C} G^{B D}\right) \partial_{B} A_{C}\right]-\left[M^{2}+c \delta(y)\right] G^{D B} A_{B}=0 .
$$

This equation can be solved by separating to the one of $A_{y}$ and $A_{\mu}$, the transverse and parallel parts to the brane. The parallel part $A_{\mu}$ is further separated to the transverse and longitudinal parts with respect to the four-dimensional momentum on the brane,

$$
A_{\mu}=A_{\mu}^{T}+A_{\mu}^{L}
$$

where $A_{\mu}^{T}=\left(\eta_{\mu \nu}-\partial_{\mu} \partial_{\nu} / \square\right) \eta^{\nu \rho} A_{\rho}$ and $A_{\mu}^{L}=\left(\partial_{\mu} \partial_{\nu} / \square\right) \eta^{\nu \rho} A_{\rho}$ with $\square=\eta^{\mu \nu} \partial_{\mu} \partial_{\nu}$. The projection-operators for $A_{\rho}$ presented here are useful in the case of non-zero eigenvalue 
for $\square$. For the case of zero-eigenvalue, this projection is not necessary since $A_{\mu}^{L}$ is absent if the gauge fixing term is not considered.

We notice that it is necessary to consider even and odd functions both for $y \rightarrow-y$ in solving Eq. (5). This is a different point from the case of the massless vector fields. Here we restrict to the following two simple cases; $A_{\mu}^{T}$ is even, i.e., $A_{\mu}^{T}(x, y)=A_{\mu}^{T}(x,|y|)$, and either $A_{\mu}^{L}$ or $A_{y}$ is odd. Namely,

$$
A_{\mu}^{L}(x, y)=\operatorname{sgn}(y) f_{\mu}(x,|y|), \quad A_{y}(x, y)=f_{y}(x,|y|),
$$

or

$$
A_{y}(x, y)=\operatorname{sgn}(y) f_{y}(x,|y|), \quad A_{\mu}^{L}(x, y)=f_{\mu}(x,|y|) .
$$

Here $\operatorname{sgn}(y)$ is defined as $\operatorname{sgn}(y)=|y| / y$ for $y \neq 0$ and $\operatorname{sgn}(y)=0$ for $y=0$. In both cases, we obtain from Eq. (5) by operating $\partial_{D}$ on it the following relation

$$
\eta^{\mu \nu} \partial_{\mu} f_{\nu}=-e^{-2|y| / L}\left(f_{y}^{\prime}-\frac{4}{L} f_{y}\right),
$$

where $f_{y}^{\prime}=d f_{y} / d|y|$. Here we solve (5) with respect to $A_{\mu}^{T}$ and $A_{y}$ firstly, then $A_{\mu}^{L}$ is obtained from (9) in terms of the $A_{y}$ solved.

Using (5) and (9), we obtain the equations for $A_{\mu}^{T}$ and $A_{y}$. In order to write these equations in the form of one-dimensional "Schrödinger equation", $A_{\mu}^{T}$ and $A_{y}$ are replaced in the form,

$$
A_{\mu}^{T}=(|z| / L+1)^{1 / 2} \hat{\psi}(x, z), \quad A_{y}=(|z| / L+1)^{5 / 2} \hat{\psi}(x, z),
$$

where $z=\operatorname{sgn}(y) L\left(e^{|y| / L}-1\right)$. Further imposing $\square \hat{\psi}(x, z)=m^{2} \hat{\psi}(x, z)$ for $\hat{\psi}(x, z)$, where $m$ denotes the four-dimensional mass of the bulk fields. Then we obtain

$$
\left[-\partial_{z}^{2}+V(z)\right] \hat{\psi}(x, z)=m^{2} \hat{\psi}(x, z),
$$

where

$$
V(z)=\frac{a}{(|z| / L+1)^{2}}-b \delta(z), \quad a=\frac{3}{4 L^{2}}+M^{2} .
$$

The parameter $b$ is given by $b=1 / L-c$ for $A_{\mu}^{T}$. While $b=-3 / L-c$ and $b=$ $-3 / L-4 M^{2} / c$ for $A_{y}$ of (7) and (8) respectively. In (11), the $x$-dependent part can be factored out, so we concentrate on its $z$-dependence hereafter.

For $A_{\mu}^{T}$, the equation (11) can be solved with $b=1 / L-c$ and the following boundary condition,

$$
\left.\partial_{z} \hat{\psi}(z)\right|_{z=0}=-\left.\frac{1}{2}(1 / L-c) \hat{\psi}(z)\right|_{z=0},
$$

which is required from the equation (111) due to the existence of the $\delta$-function in the potential. It is easy to see that this condition is consistent with the one obtained from (5) for $A_{\mu}^{T}$. Then the solution for $m>0$ is obtained as

$$
\hat{\psi}(z)=N(|z| / L+1)^{1 / 2}\left[J_{\nu}(m[|z|+L])+\alpha Y_{\nu}(m[|z|+L])\right]
$$


where $\nu=\sqrt{1+M^{2} L^{2}}, N$ denotes the normalization factor and

$$
\alpha=-\frac{m J_{\nu}^{\prime}(m L)+(1 / L-c / 2) J_{\nu}(m L)}{m Y_{\nu}^{\prime}(m L)+(1 / L-c / 2) Y_{\nu}(m L)} .
$$

But we know that this mode does not localize on the brane. It is seen from the equation (11). For $1 / L>c$ and $a>0$, we obtain the so-called volcano-type potential which is necessary for the localization of the mode given by the solution of this equation. However any mode with $m>0$ would decay into the bulk with a finite life-time as shown in [13, 15]. The modes with $m>0$ are identified with the continuous Kaluza-Klein $(\mathrm{KK})$ modes. Then the localizable and stable state is restricted to the "zero-mode" of $m=0$ 13.

The normalizable solution of zero-mode is given by

$$
\hat{\psi}(z)=N(|z| / L+1)^{1 / 2-\nu},
$$

In this case, the parameter $c$ is determined from the boundary condition (13) as,

$$
c=-\frac{2(\nu-1)}{L}
$$

Then, this mode is localized on the brane as a massless vector boson, namely as the gauge boson, when the parameter $c$ is chosen as above.

Next, we examine $A_{y}$. Its solution can be obtained similarly to the case of $A_{\mu}^{T}$ by solving (11) with $b=-3 / L-c$ and the boundary condition,

$$
\left.\partial_{z} \hat{\psi}(z)\right|_{z=0}=\left.\frac{1}{2}(3 / L+c) \hat{\psi}(z)\right|_{z=0},
$$

for the solution of (7). In the case of (8), the boundary condition is obtained by replacing the second term $c$ in the parenthesis of (18) by $4 M^{2} / c$. In any case, the explicit form of the solution could be given in a similar form to (14) with a different $\alpha$ which is determined by the boundary condition. We do not give it since it is not important here.

Interesting point to be examined for this solution is that we could expect zero-mode bound state for $b>0$ or $c<-3 / L$, where attractive $\delta$-function potential exists. The necessary condition to realize this bound state is obtained in a similar way as in the case of $A_{\mu}^{T}$. The normalizable solution of the zero-mode is given by the same form with (16) in this case also, and we obtain (17) as the condition for the case of (8). This fact seems to imply that we can observe both massless vector and scalar, which is identified with $A_{y}$ here, on the brane in this case. However scalar field can not be seen on the brane because of the factor $\operatorname{sgn}(y)$ which vanishes at $y=0$, just on the brane.

For the case of (7), the condition is obtained as follows,

$$
c=-\frac{2(\nu+1)}{L} \text {. }
$$


In this case, the value of $c$ given by (17) does not satisfy the above equations at any $\nu$, so the scalar zero-mode can not be bounded when vector zero-mode is bounded and vice versa.

The second point to be studied is how is about the bound state of the tachyonic scalar, the state with $m^{2}<0$, when the zero-mode of $A_{\mu}^{T}$ is bounded. Since only one bound state is expected for the case of $\delta$-function potential, there is no tachyonic bound state for the case of $A_{\mu}^{T}$ and $A_{y}$ for (8). While for $A_{y}$ of (7), we can expect the tachyonic bound state since the value of $b$ is smaller than that of $A_{\mu}^{T}$ or the one of $A_{y}$ for the case of (8). The values of $b$ for the latter two cases are the same, and zero-mode is bounded for both cases. If there exists a tachyonic bound state of $A_{y}$ for (]), its position at $m=i|m|$ is given by solving the following equation [13],

$$
L|m| K_{\nu-1}(L|m|)+2 K_{\nu}(L|m|)=0 .
$$

It is easy to assure that there is no solution for $|m|>0$, where both terms in the left hand side of the equation (20) are positive for any $\nu$. Then we can realize the situation where only "photon" is bounded on the brane without any tachyonic state.

As for the tachyonic bound state for $A_{\mu}^{T}$ or the one of $A_{y}$ for the case of (8), it appears when $c$ is taken as an appropriate negative value. For example, we can find it for $c=-2 / L$ and $\nu<2$. We do not consider these cases here.

The effective action for the localized zero-mode of $A_{\mu}^{T}$ can be written by denoting it as $A_{\mu}(x, y)=a_{\mu}(x) u(y)$ and substituting the solution obtained above for $u(y)$, which is given by

$$
u(y)=e^{-(\nu-1) y / L}
$$

where we set $u(0)=1$ since the normalization can be absorbed into $a_{\mu}(x)$. Then the effective action is obtained as

$$
\int d^{4} x\left(-\frac{1}{4} f_{\mu \nu} f^{\mu \nu}\right)
$$

where the suffices are contracted by $\eta_{\mu \nu}$ and $f_{\mu \nu}=\partial_{\mu} \tilde{a}_{\nu}-\partial_{\nu} \tilde{a}_{\mu}$. Here

$$
\tilde{a}_{\mu}(x)=\sqrt{\int_{0}^{\infty} d y u(y)^{2}} a_{\mu}(x)=\sqrt{\frac{L}{2(\nu-1)}} a_{\mu}(x) .
$$

The above integral with respect to $y$ is finite for $\nu>1$ or $M^{2}>0$, which is the case considered here.

In any case, we can see that the zero mode of the bulk vector is localized on the brane as a gauge boson when a quadratic form of potential of this vector is added on the brane. In the next section, we see this point from the Green function for the vector fields.

\section{Vector propagator}

In this section we analyze the Green function of massive vector in the $d+1$ dimensional AdS space to see its effective propagator observed in $d$ dimensional flat space of the 
brane. After obtaining the Green function, we go back to the case of $d=4$. We work in the brane background of the AdS metric (3).

The action integral for free massive vector $A_{M}(X)$ with external source $J_{M}(X)$ is given by

$$
S_{d+1}=S_{A d+1}-\int d^{d+1} X \sqrt{-G} G^{M N} A_{M} J_{N},
$$

where $S_{A d+1}$ is the $d+1$ dimensional version of $S_{A}$ defined by (雨). Then the Green function $\Delta_{M N}\left(X, X^{\prime}\right)$ is defined by

$$
\begin{aligned}
& D^{B N}(X) \Delta_{N P}\left(X, X^{\prime}\right)=\frac{1}{\sqrt{-G}} \delta^{B}{ }_{P} \delta^{d+1}\left(X-X^{\prime}\right), \\
& D^{B N} \Delta_{N P}= \frac{1}{\sqrt{-G}} \partial_{A}\left[\sqrt{-G}\left(G^{A M} G^{B N}-G^{A N} G^{B M}\right) \partial_{M} \Delta_{N P}\right] \\
&-\left[M^{2}+c \delta(y)\right] G^{B N} \Delta_{N P} .
\end{aligned}
$$

From this definition it follows the relation

$$
\frac{1}{\sqrt{-G}} \partial_{B}\left(\sqrt{-G}\left[M^{2}+c \delta(y)\right] G^{B N} \Delta_{N P}\right)=-\frac{1}{\sqrt{-G}} \partial_{P} \delta^{d+1}\left(X-X^{\prime}\right)
$$

as a consequence of the identity

$$
\partial_{B} \partial_{A}\left[\sqrt{-G}\left(G^{A M} G^{B N}-G^{A N} G^{B M}\right) \partial_{M} \Delta_{N P}\right]=0 .
$$

In (25) and (27), it should be understood as $\delta^{d+1}\left(X-X^{\prime}\right)=\delta^{d}\left(x-x^{\prime}\right) \delta\left(y-y^{\prime}\right)$.

Let us change variables from the coordinate $X^{M}=\left(x^{\mu}, y\right)$ to the coordinate $X^{M}=$ $\left(x^{\mu}, z\right)$, where $z=L \exp (|y| / L)$, which is different from $z$ used in the previous section. In terms of the coordinate $X^{M}=\left(x^{\mu}, z\right)$, AdS metric is written as

$$
d s^{2}=G_{M N} d X^{M} d X^{N}=\frac{L^{2}}{z^{2}}\left(\eta_{\mu \nu} d x^{\mu} d x^{\nu}+d z^{2}\right)
$$

The propagators are assumed to be

$$
\begin{aligned}
\Delta_{\mu \nu}\left(x, y ; x^{\prime}, y^{\prime}\right) & =\Delta_{\mu \nu}\left(x, z ; x^{\prime}, z^{\prime}\right), \\
\Delta_{y \nu}\left(x, y ; x^{\prime}, y^{\prime}\right) & =\operatorname{sgn}(y) \frac{z}{L} \Delta_{z \nu}\left(x, z ; x^{\prime}, z^{\prime}\right), \\
\Delta_{\mu y}\left(x, y ; x^{\prime}, y^{\prime}\right) & =\Delta_{\mu z}\left(x, z ; x^{\prime}, z^{\prime}\right) \frac{z^{\prime}}{L} \operatorname{sgn}\left(y^{\prime}\right), \\
\Delta_{y y}\left(x, y ; x^{\prime}, y^{\prime}\right) & =\operatorname{sgn}(y) \frac{z}{L} \Delta_{z z}\left(x, z ; x^{\prime}, z^{\prime}\right) \frac{z^{\prime}}{L} \operatorname{sgn}\left(y^{\prime}\right),
\end{aligned}
$$

These forms are inspired from the general coordinate transformation and the possibility considered in the previous section that $A_{y}(x, y)$ is odd function of $y$. 
The components $D^{B N}$ in the definition (25) are given by the followings;

$$
\begin{aligned}
D^{\beta \nu} & =\left(\frac{z}{L}\right)^{4}\left[\eta^{\beta \nu} D_{1}-\eta^{\alpha \nu} \eta^{\beta \mu} \partial_{\alpha} \partial_{\mu}\right]+\delta(z-L) \eta^{\beta \nu}\left(2 \partial_{z}-c\right), \\
D^{\beta z} & =-\left(\frac{z}{L}\right)^{4} \eta^{\beta \mu} \partial_{\mu}\left(\partial_{z}-\frac{d-3}{z}\right)-\delta(z-L) 2 \eta^{\beta \mu} \partial_{\mu}, \\
D^{z \nu} & =-\left(\frac{z}{L}\right)^{4} \eta^{\alpha \nu} \partial_{\alpha} \partial_{z} \\
D^{z z} & =\left(\frac{z}{L}\right)^{4}\left[\square-\frac{(M L)^{2}}{z^{2}}\right] \\
D_{1} & \equiv \partial_{z}^{2}-\frac{d-3}{z} \partial_{z}-\frac{(M L)^{2}}{z^{2}}+\square .
\end{aligned}
$$

Here, in (25), it should be understood as $B, N=\mu, z$ and $\delta^{d+1}\left(X-X^{\prime}\right)=\delta^{d}(x-$ $\left.x^{\prime}\right) \delta\left(z-z^{\prime}\right)$. As for the relation (27) reads in components as

$$
\begin{aligned}
& M^{2}\left[\eta^{\beta \nu} \partial_{\beta} \Delta_{\nu P}+\left(\partial_{z}-\frac{d-1}{z}\right) \Delta_{z P}\right]+\delta(z-L)\left(c \eta^{\beta \nu} \partial_{\beta} \Delta_{\nu P}+2 M^{2} \Delta_{z P}\right) \\
= & -\left(\frac{z}{L}\right)^{d-1} \partial_{P} \delta^{d+1}\left(X-X^{\prime}\right) .
\end{aligned}
$$

Here also it should be understood as $P=\mu, z$ and $\delta^{d+1}\left(X-X^{\prime}\right)=\delta^{d}\left(x-x^{\prime}\right) \delta\left(z-z^{\prime}\right)$.

The defining equations (25) for the Green functions are solved off brane $(z \neq L)$ as follows. (i) With the aid of relations (39), $\eta^{\beta \nu} \partial_{\beta} \Delta_{\nu P}$ are written in terms of $\Delta_{z P}$. (ii) Then closed equations are obtained for $\Delta_{z z}$ and $\Delta_{z \rho}$ by themselves and they are easily solved. (iii) As for $\Delta_{\alpha z}$ and $\Delta_{\alpha \rho}$, the equations become inhomogeneous equation again with the aid of relations (39). Since the inhomogeneous terms for $\Delta_{\alpha z}\left(\Delta_{\alpha \rho}\right)$ is already solved $\Delta_{z z}\left(\Delta_{z \rho}\right)$ and $\delta\left(z-z^{\prime}\right)$, they are solved with as yet undetermined homogeneous solutions. (iv) The undetermined homogeneous solutions are determined in such a way that $\Delta_{M N}$ satisfy the relation (39). The resulting solutions are given by the followings;

$$
\begin{aligned}
\Delta_{\alpha \rho}= & \Delta_{\alpha \rho}^{T}+\Delta_{\alpha \rho}^{L}, \\
\Delta_{\alpha \rho}^{T}= & \left(\frac{z z^{\prime}}{L^{2}}\right)^{d / 2-1} \int \frac{d^{d} p}{(2 \pi)^{d}} e^{i p\left(x-x^{\prime}\right)}\left(\eta_{\alpha \rho}-\frac{p_{\alpha} p_{\rho}}{p^{2}}\right) \hat{\Delta}_{1}\left(p, z, z^{\prime}\right), \\
\Delta_{\alpha \rho}^{L}= & -\frac{1}{M^{2}}\left(\frac{z z^{\prime}}{L^{2}}\right)^{d / 2} \int \frac{d^{d} p}{(2 \pi)^{d}} e^{i p\left(x-x^{\prime}\right)} \frac{p_{\alpha} p_{\rho}}{p^{2}}\left[\frac{L}{z^{\prime}} \delta\left(z-z^{\prime}\right)\right. \\
& \left.+\left(\partial_{z}-\frac{d / 2-1}{z}\right)\left(\partial_{z}^{\prime}-\frac{d / 2-1}{z^{\prime}}\right) \hat{\Delta}_{2}\left(p, z, z^{\prime}\right)\right], \\
\Delta_{\alpha z=} & -\frac{1}{M^{2}} \partial_{\alpha}\left(\partial_{z}-\frac{d-1}{z}\right) \Delta_{2}\left(X, X^{\prime}\right), \\
\Delta_{z \rho}= & -\frac{1}{M^{2}}\left(\partial_{z}^{\prime}-\frac{d-1}{z^{\prime}}\right) \partial_{\rho}^{\prime} \Delta_{2}\left(X, X^{\prime}\right),
\end{aligned}
$$




$$
\Delta_{z z}=-\frac{1}{M^{2}}\left(\frac{z z^{\prime}}{L^{2}}\right)^{\frac{d-1}{2}} \delta^{d+1}\left(X-X^{\prime}\right)+\frac{\square}{M^{2}} \Delta_{2}\left(X, X^{\prime}\right),
$$

where $\partial_{z}^{\prime}\left(\partial_{\alpha}^{\prime}\right)$ denotes differentiation with respect to $z^{\prime}\left(x^{\prime \alpha}\right)$. The scalar-type propagators $\Delta_{1}\left(X, X^{\prime}\right)$ and $\Delta_{2}\left(X, X^{\prime}\right)$ are defined by the followings;

$$
\begin{aligned}
& D_{1}(X) \Delta_{1}\left(X, X^{\prime}\right)=\left(\frac{z^{\prime}}{L}\right)^{d-3} \delta^{d+1}\left(X-X^{\prime}\right) \\
& D_{2}(X) \Delta_{2}\left(X, X^{\prime}\right)=\left(\frac{z^{\prime}}{L}\right)^{d-1} \delta^{d+1}\left(X-X^{\prime}\right) \\
& D_{2} \equiv \partial_{z}^{2}-\frac{d-1}{z} \partial_{z}-\frac{-(d-1)+(M L)^{2}}{z^{2}}+\square \\
& \Delta_{1}\left(X, X^{\prime}\right)=\left(\frac{z z^{\prime}}{L^{2}}\right)^{d / 2-1} \int \frac{d^{d} p}{(2 \pi)^{d}} e^{i p\left(x-x^{\prime}\right)} \hat{\Delta}_{1}\left(p, z, z^{\prime}\right) \\
& \Delta_{2}\left(X, X^{\prime}\right)=\left(\frac{z z^{\prime}}{L^{2}}\right)^{d / 2} \int \frac{d^{d} p}{(2 \pi)^{d}} e^{i p\left(x-x^{\prime}\right)} \hat{\Delta}_{2}\left(p, z, z^{\prime}\right) \\
& {\left[\partial_{z}^{2}+\frac{1}{z} \partial_{z}+\left(q^{2}-\frac{\nu^{2}}{z^{2}}\right)\right] \hat{\Delta}_{i}\left(p, z, z^{\prime}\right)=\frac{L}{z^{\prime}} \delta\left(z-z^{\prime}\right)} \\
& q^{2}=-p^{2}, \quad \nu \equiv\left[(d / 2-1)^{2}+(M L)^{2}\right]^{1 / 2}
\end{aligned}
$$

It should be noted that the $\delta$-function singularity is canceled by the second term in the square bracket of (42) and that $\hat{\Delta}_{1}$ only contributes to transverse parts while $\hat{\Delta}_{2}$ contributes to longitudinal parts. It is not difficult to directly verify that (40) (45) satisfy the relation (39) and the defining equation (25) by using the following relations;

$$
\begin{aligned}
& \Delta_{i}\left(X, X^{\prime}\right)=\Delta_{i}\left(X^{\prime}, X\right), \quad(i=1,2) \\
& \hat{\Delta}_{i}\left(p, z, z^{\prime}\right)=\hat{\Delta}_{i}\left(p, z^{\prime}, z\right), \quad(i=1,2) \\
& D_{1}\left(\partial_{z}-\frac{d-1}{z}\right)=\left(\partial_{z}-\frac{d-3}{z}\right) D_{2}-\frac{2}{z} \square .
\end{aligned}
$$

Having obtained the Green functions in the bulk, we now proceed to impose boundary conditions on them on the brane. They are obtained by matching $\delta(z-L)$ in the defining equation (25) and the relation (39);

$$
\begin{aligned}
& \left.2 \partial_{z} \Delta_{\alpha \rho}^{T}\right|_{z=L}-\left.c \Delta_{\alpha \rho}^{T}\right|_{z=L}=0 \\
& \left.2 \partial_{z} \Delta_{\alpha \rho}^{L}\right|_{z=L}-\left.c \Delta_{\alpha \rho}^{L}\right|_{z=L}-\left.2 \partial_{\alpha} \Delta_{z \rho}\right|_{z=L}=0, \\
& \left.c \eta^{\beta \nu} \partial_{\beta} \Delta_{\nu \rho}^{L}\right|_{z=L}+\left.2 M^{2} \Delta_{z \rho}\right|_{z=L}=0 \\
& \left.c \eta^{\beta \nu} \partial_{\beta} \Delta_{\nu z}^{L}\right|_{z=L}+\left.2 M^{2} \Delta_{z z}\right|_{z=L}=0 .
\end{aligned}
$$

It turns out that it is convenient for our purpose to set as

$$
c=-\frac{2(\nu-d / 2+1)}{L}
$$


which coincide with (17) when $d=4$. This value is chosen in such a way that the leading pole of $\Delta_{\mu \nu}^{T}$ is massless (see $\left.(63) \sim 65\right)$ ).

First we discuss about a solution for $\hat{\Delta}_{1}$. The boundary condition (56) is obtained as a consequence of the potential on the brane and it differs from the Neumann condition. It is a mixed boundary condition of Dirichlet type and of Neumann type, which is briefly commented in the previous paper [13] and is employed in [16]. As we have imposed the boundary condition (56) on the brane, the procedure to obtain the propagator is parallel to [14]. The result is given by

$$
\hat{\Delta}_{1}\left(p, z, z^{\prime}\right)=\frac{i \pi L}{2}\left[\frac{J_{\nu-1}(q L)}{H_{\nu-1}^{(1)}(q L)} H_{\nu}^{(1)}(q z) H_{\nu}^{(1)}\left(q z^{\prime}\right)-J_{\nu}\left(q z_{<}\right) H_{\nu}^{(1)}\left(q z_{>}\right)\right],
$$

where $z_{>}\left(z_{<}\right)$denotes the greater (lesser) of $z$ and $z^{\prime}$. A case that is of particular interest here is that where the arguments of $\Delta_{\mu \nu}^{T}\left(x, z ; x, z^{\prime}\right)$ is on the brane, $z=z^{\prime}=L$. In this case, the propagator is expressed as

$$
\begin{aligned}
\Delta_{\alpha \rho}^{T}\left(x, L ; x^{\prime}, L\right) & =\int \frac{d^{d} p}{(2 \pi)^{d}} e^{i p\left(x-x^{\prime}\right)}\left(\eta_{\alpha \rho}-\frac{p_{\alpha} p_{\rho}}{p^{2}}\right) \frac{1}{q} \frac{H_{\nu}^{(1)}(q L)}{H_{\nu-1}^{(1)}(q L)} \\
& =\int \frac{d^{d} p}{(2 \pi)^{d}} e^{i p\left(x-x^{\prime}\right)}\left(\eta_{\alpha \rho}-\frac{p_{\alpha} p_{\rho}}{p^{2}}\right)\left[\frac{2(\nu-1)}{q^{2} L}-\frac{1}{q} \frac{H_{\nu-2}^{(1)}(q L)}{H_{\nu-1}^{(1)}(q L)}\right] .
\end{aligned}
$$

From this result, some interesting features are observed. Hereafter we consider the case of $d=4$.

As in Ref. [14], $\Delta_{\mu \nu}^{T}\left(x, L ; x^{\prime}, L\right)$ is split into the sum of the 4-dimensional massless propagator and the exchange of the Kaluza-Klein states;

$$
\begin{aligned}
\Delta_{\alpha \rho}^{T}\left(x, L ; x^{\prime}, L\right) & =\frac{2(\nu-1)}{L} \Delta_{\alpha \rho}^{T(0)}\left(x, x^{\prime}\right)+\Delta_{\alpha \rho}^{T(K K)}\left(x, x^{\prime}\right) \\
\Delta_{\alpha \rho}^{T(0)}\left(x, x^{\prime}\right) & =\int \frac{d^{4} p}{(2 \pi)^{4}} e^{i p\left(x-x^{\prime}\right)}\left(\eta_{\alpha \rho}-\frac{p_{\alpha} p_{\rho}}{p^{2}}\right) \frac{1}{q^{2}} \\
\Delta_{\alpha \rho}^{T(K K)}\left(x, x^{\prime}\right) & =-\int \frac{d^{4} p}{(2 \pi)^{4}} e^{i p\left(x-x^{\prime}\right)}\left(\eta_{\alpha \rho}-\frac{p_{\alpha} p_{\rho}}{p^{2}}\right) \frac{1}{q} \frac{H_{\nu-2}^{(1)}(q L)}{H_{\nu-1}^{(1)}(q L)} .
\end{aligned}
$$

The propagator $\Delta^{T(0)}\left(x, x^{\prime}\right)$ represents the localized massless state in the 4-dimensional brane. This localized mode is precisely the gauge boson (photon) trapped on the brane. This is a consequence of the choice (60) as previously noted. The leading part of the summation of the KK exchanges gives $1 / r^{3}$ potential for $\nu>2$ as in the case of the gravity while it gives $1 / r^{\nu+1}$ potential for $1<\nu<2$. Thus we have succeeded to trap a photon on the brane as a leading massless mode of vector. In order to obtain this result the potential on the brane has played an important role.

On the other hand, boundary conditions $(57) \sim(59)$ are satisfied if the following boundary condition for $\hat{\Delta}_{2}$ is satisfied;

$$
\left.\left(\partial_{z}-\frac{d / 2-1}{z}-\frac{2 M^{2}}{c}\right) \hat{\Delta}_{2}\left(p, z, z^{\prime}\right)\right|_{z=L}=0 . \quad\left(z^{\prime}>L\right)
$$


Therefore $\hat{\Delta}_{2}$ has a nontrivial solution for the present case in contrast to the case when $\Delta_{M N}$ are even functions of $y$ for all $M, N=\mu, y$. In latter case there is no solution for $\hat{\Delta}_{2}$. With the value of $c$ given by (60), the above boundary condition (66) reads as

$$
\left.\left(\partial_{z}-\frac{\nu}{L}\right) \hat{\Delta}_{2}\left(p, z, z^{\prime}\right)\right|_{z=L}=0 . \quad\left(z^{\prime}>L\right)
$$

This boundary condition is the same as the one for $\hat{\Delta}_{1}$ so that

$$
\hat{\Delta}_{2}\left(p, z, z^{\prime}\right)=\hat{\Delta}_{1}\left(p, z, z^{\prime}\right)
$$

Thus $\Delta_{2}\left(X, X^{\prime}\right)$ is split into the sum of 4-dimensional massless propagator and the exchange of the Kaluza-Klein states as well as $\Delta_{1}\left(X, X^{\prime}\right)$. However the massless pole of $\Delta_{2}$ in $\Delta_{y \nu}, \Delta_{\mu y}$, and $\Delta_{y y}$ cannot be seen on the brane because of the factor $\operatorname{sgn}(y)$ which vanishes at $y=0$, just on the brane. This result is consistent with the consideration in the previous section.

\section{Summary}

We have examined the massive vector field in the AdS background, in which the Randall-Sundrum three-brane is embedded. We find the localization of the leading massless mode of the transverse part of the massive vector on the brane. This result is assured by both wave-function analysis and propagator analysis. The leading massless mode is nothing but a gauge boson (photon) as is seen from the effective action (22). In contrast to [10], the mass of the trapped gauge boson is strictly vanishing in our analysis. For the purpose of obtaining the above conclusion, the potential on the brane, which take forms of tachyonic mass terms, play an essential role. Although the origin of the potentials on the brane is obscure at present, we would like to point out some resemblance of them to the potential on the brane for scalar considered in [11], where tachyonic mass term is contained in the Higgs-type potential.

On the other hand, it is necessary for getting nontrivial solutions for the longitudinal mode $\left(A_{\mu}^{L}\right)$ and the one transverse to the brane $\left(A_{y}\right)$ to assume opposite transformation properties under $y \rightarrow-y$. If we assumed that $A_{\mu}^{L}$ and $A_{y}$ are both even functions of $y$, there would be no nontrivial solutions. In the propagator approach, the case of even $A_{\mu}$ and odd $A_{y}$ is examined and the above statement is assured. This situation is in contrast to the case of massless vector where odd function is not necessary.

In addition to the potential on the brane, the mass term of the bulk vector is necessary to obtain the normalizable wave-function. The mechanism proposed here is curious in the sense that the broken gauge symmetry in the bulk is restored on the brane. It could be considered as a kind of inverse Higgs mechanism into the reduced dimensions.

It will be interesting to study the case including the charged particles [17, 18], since our model could provide concrete form of z-dependent wave-function and propagator of the gauge fields. We will give the discussion related to these in the future. 


\section{References}

[1] L. Randall and R. Sundrum, Phys. Rev. Lett. 83 (1999) 3370, (hep-ph/9905221).

[2] L. Randall and R. Sundrum, Phys. Rev. Lett. 83 (1999) 4690, (hep-th/9906064).

[3] B. Bajc and G. Gabadadze, Phys. Lett. B474 (2000) 282, (hep-th/9912232).

[4] Y. Grossman and M. Neubert, Phys. Lett. B474 (2000) 361, (hep-ph/9912408).

[5] S. Chang, J. Hisano, H. Nakano, N. Okada and M. Yamaguchi, Phys. Rev. D62 (2000) 084025, (hep-ph/9912498).

[6] G. Dvali and M. Shifman, Phys. Lett. B396 (1997) 64 [Erratum-ibid. B407 (1997) 452], (hep-th/9612128).

[7] G. Dvali, G. Gavadadze and M. Shifman, Phys. Lett. B497 (2001) 271, (hep-th/0010071).

[8] P. Dimopoulos, K. Farakos, A. Kehagias and G. Koutsoumbas, "Lattice Evidence for Gauge Field Localization on a Brane", hep-th/0007079.

[9] M.J. Duff, J.T. Liu, and W.A. Sabra, "Localization of supergravity on the brane", hep-th/0009212.

[10] I. Oda, "A New Mechanism for Trapping of Photon", hep-th/0103052.

[11] W.D. Goldberger and M.B. Wise, Phys. Rev. Lett. 83 (1999) 4922, (hep-ph /9907447).

[12] H. Georgi, A. K. Grant and G. Hailu, Phys. Lett. B506 (2001) 207, (hep-ph /0012379).

[13] R. Ghoroku and A. Nakamura, "Stability of Randall-Sundrum brane-world and tachyonic scalar", hep-th/0103071.

[14] S.B. Giddings, E. Katz and L. Randall, JHEP 03 (2000) 023, (hep-th/0002091).

[15] S.L. Dubovski, V.A. Rubakov and P.G. Tinyakov, Phys. Rev. D62 (2000) 105011, (hep-th/0006046).

[16] M. Mintchev and L. Pilo, Nucl. Phys. B592 (2001) 219, (hep-th/0007002).

[17] S.L. Dubovsky, V.A. Rubakov, P.G. Tinyakov, JHEP 08 (2000) 041, (hep-th /0007179).

[18] S.L. Dubovsky, V.A. Rubakov, "On models of gauge field localization on a brane", hep-th/0105243. 